\title{
Manejo pré-abate de transporte e banho sobre a incidência de mortalidade de frangos de corte
}

\section{Preslaughter transportation and shower management on broiler chicken Dead on Arrival (DOA) incidence}

\author{
Janaina Aparecida Oliveira Silva'; Gislaine Silveira Simões ${ }^{2}$; Alessandro Rossa ${ }^{3}$; \\ Alexandre Oba ${ }^{4}$; Elza Iouko Ida ${ }^{5}$; Massami Shimokomaki ${ }^{6 *}$
}

\begin{abstract}
Resumo
O objetivo deste trabalho foi avaliar o efeito da distância de transporte e do banho após o carregamento sobre a mortalidade de frangos na chegada ao abatedouro, denominada Mortalidade na Chegada, do ingles Dead on Arrival - DOA. O estudo foi conduzido no inverno e no verão de 2008 em abatedouro comercial. No inverno, as distâncias de transporte foram de 15 e $57 \mathrm{~km}$, e no verão foram de 15 e 55 $\mathrm{km}$, sendo os experimentos realizados em duplicata. No verão, para avaliar o efeito do banho sobre os frangos após o carregamento no caminhão, foram avaliados dois lotes em cada distância, lote sem banho (LSB) e lote com banho (LCB). Pelos resultados verificou-se que, no inverno o DOA não foi alterado pela distância de transporte. Enquanto que no verão, o DOA variou de $0,16 \%$ a $0,27 \%$ nos lotes LSB e de $0,12 \%$ a $0,17 \%$ nos lotes LCB. No verão, foram observados menores valores de DOA nos lotes LCB nas duas distâncias de transporte indicando que o banho após o carregamento reduziu a mortalidade dos frangos durante o transporte.
\end{abstract}

Palavras-chave: Banho. Conforto térmico. Manejo pré-abate.

\begin{abstract}
The aim of this work was to evaluate the influence of the management conditions and distance to a commercial plant on chicken broilers Dead on Arrival (DOA). This work was conducted in the winter and summer seasons of 2008 in a commercial plant. In the winter season, the transport distances were 15 and $57 \mathrm{~km}$, and in the summer season the distances were 15 and $55 \mathrm{~km}$ from the farm to the slaughterhouse and the experiments were carried out in duplicate. In the summer, to evaluate the effect of water bath over the birds before the actual transportation, a group of two trucks were submitted to a water bath (GWthB) and the other without water bath treatment (GWoutB). The results showed that in the winter, the DOA was not influenced to distance of transport. In the summer, the DOA values for GWthB group varied from 0.12 to $0.17 \%$ while for the GWoutB the DOA values ranged from 0.16 to $0.27 \%$ indicating that the bath treatment after broilers truck loading and before transportation reduced broiler chicken mortality.

Key words: Bath. Preslaughter. handling. Thermal comfort.
\end{abstract}

Graduanda em Zootecnia. Departamento de Zootecnia. Centro de Ciências Agrárias. Universidade Estadual de Londrina, UEL. E-mail: janazoo@hotmail.com

Doutoranda em Ciência de Alimentos. Departamento de Ciência e Tecnologia de Alimentos. Centro de Ciências Agrárias. Universidade Estadual de Londrina, UEL. E-mail: gixlaine@yahoo.com.br

Médico Veterinário, Universidade Estadual de Londirna, UEL. E-mail: alesrossa@hotmail.com

Prof. Dr. Departamento de Zootecnia. Centro de Ciências Agrárias. Universidade Estadual de Londrina, UEL. E-mail: oba@uel. br

Prof $^{\mathrm{a}}$. Dr ${ }^{\mathrm{a}}$ Departamento de Ciência e Tecnologia de Alimentos, Centro de Ciências Agrárias. Universidade Estadual de Londrina, UEL. Londrina, PR. E-mail: elida@uel.br

Prof. Dr. Departamento de Ciência e Tecnologia de Alimentos, Centro de Ciências Agrárias. Universidade Estadual de Londrina, UEL. Londrina, PR. E-mail: mshimo@uel.br

* Autor para correspondência

Recebido para publicação 20/10/2009 Aprovado em 09/05/2011 


\section{Introdução}

O tema bem-estar animal faz parte cada vez mais na consciência pública dos países desenvolvidos. Esse fato ocorre ao mesmo tempo em que há demanda por maior segurança alimentar e outras modificações de preferências pelo consumidor. A pluralidade da demanda dos consumidores e suas preocupações com o bem-estar animal de um lado, e as opções dos produtores nos diversos tipos de criações e negócios, de outro, conduzem a uma aparente mudança nos sistemas de criação e no cuidado nas etapas de manejo pré-abate, tendo como objetivo conciliar o bem-estar animal a uma produção sustentável.

Diversos fatores relacionados ao bem-estar de frangos, tais como, lesões na carcaça, estresse fisiológico e elevados índices de mortalidade estão associados principalmente ao manejo pré-abate e ao transporte das aves (NICOL; SCOTT, 1990, ARISTIDES, et al., 2007).

As estações do ano, as elevadas temperaturas e umidades relativas contribuem efetivamente para o estresse térmico durante todas as operações préabate. O Brasil é um país de clima tropical e os maiores problemas se relacionam 'a mortalidade das aves no verão.

Durante o transporte, os frangos ficam expostos a uma variedade de agentes estressores, como aceleração, vibração, impactos, jejum alimentar e hídrico, mistura social, perturbações e ruídos, além do microambiente térmico que se forma entre as aves (MITCHELL; KETTLEWELL, 1998; NICOL; SCOTT, 1990, SIMÕES et al., 2009a, 2009b). O transporte causa desde leve desconforto, com alterações na qualidade final da carne, até a morte dos animais. Estudos no Reino Unido indicaram que as mortes no transporte de aves são atribuídas principalmente ao estresse e podem atingir uma taxa de $40 \%$, sendo que esta mortalidade aumenta com a distância percorrida (WARRIS; BEVIS; BROWN, 1990).

Além da distância de transporte, outros fatores podem contribuir com o aumento da mortalidade, como a condição de saúde do animal, estresse térmico, injúrias e traumas ocorridos nas etapas de apanha e carregamento dos frangos.

A criação intensiva de frangos de corte em grande número e em locais geograficamente dispersos requer que os mesmos sejam transportados para o abatedouro em caminhões via rodovia, muitas vezes por longas distâncias.

Em recentes experimentos, Simões et al. (2009a) e Langer et al. (2010) verificaram que as distâncias de transporte e a aplicação de banho imediatamente antes do transporte interferem na qualidade final da carne, visto que em distâncias curtas a aplicação do banho proporcionaram menores ocorrências de carnes PSE. Esse fato traz importantes informações às indústrias, uma vez que os filés de frango que apresentam as características de cor pálida, textura mole e baixa capacidade de retenção de água, tornam-se impróprias para o consumo (OLIVO; SHIMOKOMAKI, 2006). Assim no Brasil, as empresas avícolas passaram a recomendar como prática de manejo pré-abate no verão, a aplicação do banho de água sobre os frangos, logo após o carregamento, para reduzir a temperatura do microambiente durante o transporte. Além disso, Simões et al. (2009a, 2009b) verificaram que a localização dos frangos no caminhão de transporte influencia na ocorrência de PSE. Sabe-se, portanto, que muitos são os fatores que contribuem com o estresse das aves durante as etapas de pré-abate e que a intensidade destes efeitos determina o nível elevado de perdas por mortalidade na chegada (DOA, do inglês Dead On Arrival) ao abatedouro.

No Brasil, considerando a relevância de pesquisas sobre o manejo pré-abate e transporte de frangos, verifica-se a importância de estudos que mostrem os prejuízos decorrentes de práticas de manejo inadequadas. Assim, este trabalho teve a finalidade de avaliar o efeito da distância de transporte e da aplicação do banho antes do transporte do aviário até o abatedouro sobre a mortalidade dos frangos na 
chegada (DOA) ao abatedouro.

\section{Material e Métodos}

O estudo foi conduzido no ano de 2008, nas estações de inverno e verão em um abatedouro localizado na região Oeste do Paraná, com frangos de linhagem comercial, sexagem mista e 47 dias de idade média de abate, com peso médio de $2,7 \mathrm{~kg} /$ ave.

No inverno, as distâncias de transporte do aviário ao abatedouro foram de 15 e $57 \mathrm{~km}$. O estudo foi realizado em duplicata, com um total de 04 caminhões e aproximadamente 4.000 frangos transportados em cada caminhão, sendo em média 09 frangos por caixa, que corresponde a aproximadamente $488 \mathrm{~cm}^{2} /$ frango.

No verão, as distâncias de transporte foram de 15 e $55 \mathrm{~km}$ do aviário até o abatedouro. Para avaliar o efeito da aplicação do banho de água sobre os frangos logo após o carregamento, foram utilizados 08 caminhões, sendo 04 caminhões para cada distância, os quais foram divididos em dois lotes, LSB que não recebeu o banho após o carregamento e LCB que recebeu o banho. Cada caminhão transportou aproximadamente 3.800 aves sendo em média 07 frangos por caixa, que corresponde a aproximadamente a $627 \mathrm{~cm}^{2} /$ frango. A Figura 1 apresenta a aplicação do banho de água sobre os frangos no caminhão antes do transporte.

Chegando ao abatedouro, tanto no inverno, quanto no verão, todos os frangos permaneceram em galpão de espera com ventilação forçada e aspersão de água por aproximadamente 60 minutos. A mortalidade dos frangos nos dois experimentos foi quantificada no momento da pendura das aves na plataforma de recepção, sendo assim denominada de Morte na Chegada ao abatedouro, ou DOA (Dead on Arrival).

Foi aplicado o teste de qui-quadrado entre as diferentes distâncias de transporte, entre o fornecimento de banho dentro da mesma distância e em distâncias diferentes.
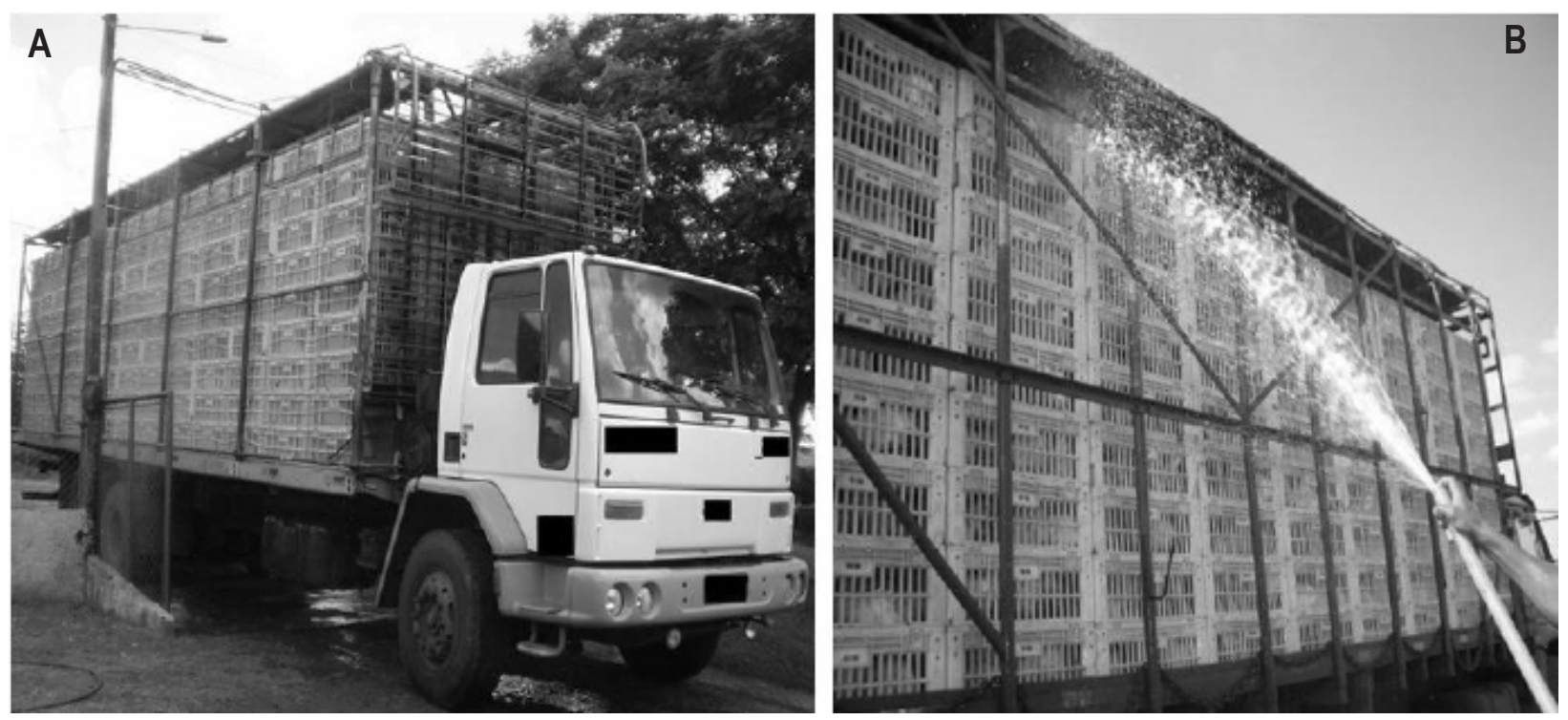

Figura 1. Aplicação do banho de água sobre os frangos no caminhão antes do transporte. A - Método com portal na saída do aviário. B - Método manual com mangueira. 


\section{Resultados e Discussão}

A Tabela 1 apresenta a DOA no inverno para as distâncias de 15 e $57 \mathrm{~km}$. Observa-se através do teste qui-quadrado que as diferentes distâncias de transporte não influenciaram $(\mathrm{P}>0,05)$ a mortalidade dos frangos, contrariando Vecerek et al. (2006), que encontraram maiores taxas de mortalidade em frangos transportados por maiores distâncias.

Os índices de mortalidade verificados neste trabalho estão dentro dos limites aceitáveis de acordo com Olivo e Shimokomaki (2001) que recomendaram $0,20 \%$ como um valor aceitável de mortalidade para as condições brasileiras.

Tabela 1. Mortalidade (DOA) dos frangos transportados no inverno nas distâncias de 15 e 57 km do aviário ao abatedouro.

\begin{tabular}{cccc}
\hline Distâncias $(\mathbf{k m})$ & Total de frangos* $^{*}$ & DOA(n) & DOA (\%) \\
\hline 15 & 7922 & 13 & 0,16 \\
57 & 7630 & 15 & 0,20 \\
\hline
\end{tabular}

$\mathrm{n}=$ número de frangos mortos

*Total de frangos em dois caminhões de transporte.

A Tabela 2 apresenta a DOA no verão dos lotes LSB e LCB transportados por 15 e $55 \mathrm{~km}$.

A DOA dos frangos do LCB transportados pelas distâncias de 15 e $55 \mathrm{~km}$ foi de 0,12 e $0,15 \%$, respectivamente, enquanto o LSB apresentou a DOA de 0,16 e $0,27 \%$, respectivamente. Através do teste de qui-quadrado, pode-se observar que a distancia de transporte influenciou significativamente a $13,33 \%$ quando foi considerado o tratamento banho e a $11,90 \%$ quando não foi considerado o tratamento banho, sendo que quanto maior a distância de transporte, maior foi a incidência de DOA observada.

Tabela 2. Mortalidade (DOA) dos frangos dos lotes LSB e LCB transportados no verão nas distâncias de 15 e $55 \mathrm{~km}$ do aviário ao abatedouro.

\begin{tabular}{ccccc}
\hline Distância $(\mathbf{k m})$ & Lote & Total de frangos* & DOA (n) & DOA (\%) \\
\hline 15 & LSB & 7616 & 12 & 0,16 \\
15 & LCB & 7570 & 09 & 0,12 \\
55 & LSB & 7376 & 20 & 0,27 \\
55 & LCB & 7760 & 12 & 0,15 \\
\hline
\end{tabular}

$\mathrm{n}=$ número de frangos mortos;

$\mathrm{LSB}=$ lote que não recebeu banho de água sobre os frangos;

$\mathrm{LCB}=$ lote que recebeu banho de água sobre os frangos;

*Total de frangos em dois caminhões de transporte. 
Estes resultados mostram que nas condições climáticas de verão do oeste do Paraná, as distâncias de transporte estudadas influenciaram na mortalidade das aves. Resultados semelhantes foram observados no Reino Unido, em trabalho realizado por Warris et al. (1992), que observaram DOA de $0,16 \%$ em transporte por menos de 4 horas, enquanto que no transporte por mais de 4 horas, a ocorrência de DOA foi de 0,28\%. Na Europa Oriental, Vecerek et al. (2006), relataram valores médios de $0,25 \%$ e verificaram que quanto maior o período de transporte, maior a porcentagem de DOA.

Ao avaliar o manejo pré-abate de banho antes do transporte, pode-se observar que este procedimento foi significativo a $11,10 \%$, reduzindo a mortalidade das aves, independentemente da distancia percorrida.

Os resultados de DOA em ambas as distâncias, foram menores do que os relatados por pesquisadores de outros países. Por exemplo, Nidjam et al. (2004) em estudos na Holanda, encontraram uma média de $0,46 \%$ de mortalidade de frangos e Petracci et al. (2006), na Itália, encontraram valores de DOA, de $0,47,0,52$ e $1,62 \%$ em frangos, perus e galinhas poedeiras, respectivamente.

Estes números, apesar da sua limitação por ter sido representativos de apenas um verão brasileiro, mostram que o manejo pré-abate desenvolvido na região oeste do Paraná, é eficiente, visto que mesmo em condições climáticas quentes, que teoricamente seria mais prejudicial à homeostase animal, os números de DOA foram menores. Para corroborar com esses resultados, Oba et al. (2009), verificaram que em condições dramáticas, no período mais quente do dia $\left(33^{\circ} \mathrm{C}\right)$, sem banho e transportados somente 'a baixa velocidade, em estradas de terra, por período de 180 minutos e descanso ao abate de 90 minutos, foram altamente susceptíveis ao estresse térmico, provocando maior mortalidade, chegando ao máximo de DOA, de 57,0\%.

Levando em consideração que os resultados relatados nesse trabalho foram obtidos em linha comercial, ao nosso conhecimento, são os primeiros a serem divulgados no país. Sendo o Brasil, provavelmente, o único país que rotineiramente nos meses de verão adota o banho sobre os frangos antes do transporte, esta pesquisa mostra a importância deste manejo pré-abate na manutenção do conforto térmico dos frangos no verão, com conseqüente diminuição de DOA.

Há possibilidade de se avaliar o prejuízo devido aos valores de DOA para as empresas. Assim, considerando o abate diário de 300.000 aves no estabelecimento onde a pesquisa foi realizada, os índices de mortalidade verificados no verão (Tabela 2), representariam uma perda mínima de 360 e máxima de 810 frangos diários. Com o custo de $\mathrm{R} \$ 1,75$ para cada quilograma de frango vivo (cotação de 09/10/2010), estes valores significam em termos econômicos um prejuízo de no mínimo $\mathrm{R} \$ 1.701,00 /$ dia e máximo de $\mathrm{R} \$ 3.827,50 /$ dia ao abatedouro. Portanto, em avicultura de corte, devido aos números exorbitantes, investir em práticas de manejo que diminuam a mortalidade, como o banho sobre as aves no verão, é uma alternativa viável para o abatedouro.

\section{Conclusões}

Pode-se concluir que maiores distâncias de transporte aumentam a mortalidade dos frangos e o manejo pré-abate do banho no verão reduz esta problemática.

\section{Referências}

ARISTIDES, L. G. A.; DOGNANI, R.; LOPES, C. F.; SILVA, L. G. S.; SHIMOKOMAKI, M. Diagnósticos de condenações que afetam a produtividade da carne de frangos brasileira. Revista Nacional da Carne, São Paulo, v. 22, n. 368, p. 22-28, 2007.

LANGER, R. O. S.; SIMÕES, G. S.; SOARES, A. L.; OBA, A.; ROSSA, A.; SHIMOKOMAKI, M.; IDA, E. I. Broiler transportation conditions in a brazilian commercial line and the occurrence of breast PSE (Pale, Soft, Exudative) meat and DFD-like (Dark, Firm, 
Dry) meat. Brazilian Archives Biology and Technology, Curitiba, v. 53, n. 5, p. 1161-1167, 2010.

MITCHELL, M. A.; KETTLEWELL, P. J. Physiological stress and welfare of broiler chickens in transit: solutions not problem! Poultry Science, Champaign, v. 77, n. 12, p. 1803-1814, 1998.

NICOL, C. J.; SCOTT, G. B. Pre-slaughter handling and transport of broiler-chickens. Applied Animal Behaviour Science, Amsterdam, v. 28, n. 1/2, p. 57-73, 1990.

NIDJAM,E.;ARENS,P.;LAMBOOJI,E.;DECUYPERE, E.; STEGEMAN, J. A. Factors influencing bruises and mortality of broilers during catching, transport and lairage. Poultry Science, Champaign, v. 83, n. 9, p. 16101615, 2004.

OBA, A.; ALMEIDA, M.; PINHEIRO, J. W.; IDA, E. I. I.; MARCHI, D. F.; SOARES, A. L.; SHIMOKOMAKI, M. Management of transport and lairage conditions on broiler chicken breast meat qualities and DOA (Death On Arrival). Brazilian Archives Biology and Technology, Curitiba, v. 52, p. 205-211, 2009. Special number.

OLIVO, R.; SHIMOKOMAKI, M. Carne PSE em aves. In: SHIMOKOMAKI, M. et al. (Ed.). Atualidades em ciência e tecnologia de carnes. São Paulo: Varela, p. 95103, 2006.

Carnes: no caminho da pesquisa. Cocal do Sul: IMPRINT, 2001. 155 p.

PETRACCI, M.; BIANCHI, M.; CAVANI,C.; GASPARI, P.; LAVAZZA, A. Preslaughter mortality in broiler chickens turkeys and spent hens during commercial slaughtering. Poultry Science, Champaign, v. 85, n. 9, p. 1660-1664, 2006.

SIMÕES, G. S.; OBA, A.; MATSUO, T.; ROSSA, A.; SHIMOKOMAKI, M.; IDA, E. I. Vehicle thermal microclimate evaluation during brazilian summer broiler transport and the occurrence of PSE (Pale, Soft, Exudative) meat. Brazilian Archives Biology and Technology, Curitiba, v. 52, p. 195-204, 2009a. Special number.

SIMÕES, G. S.; ROSSA, A.; OBA, A.; MATSUO, T.; SHIMOKOMAKI, M.; IDA, E. I. Transporte e ocorrência de PSE (Pale, Soft, Exudative) e a-DFD (Dark, Firm, Dry) em filés de peito de frango durante o Inverno. Revista Nacional da Carne, São Paulo, v. 23, n. 383, p. 20-30, 2009b.

VECEREK, V.; GRBALOVA, S.; VOSLAROVA, E.; JANACKOVA, B.; MALENA, M. Effects of travel distance and the season of the year on death rates of broilers transported to poultry processing plants. Poultry Science, Champaign, v. 85, n. 11, p. 1881-1884, 2006.
WARRIS, P. D.; BEVIS, E. A.; BROWN, S. N. Time spent by broiler chickens in transit to processing plants. Veterinary Record, London, v. 127, n. 25/26, p. 617-619, 1990.

WARRIS, P. D.; BEVIS, E. A.; BROWN, S. N.; EDWARDS, J. E. Longer journeys to processing plants are associated with higher mortality and broiler-chickens. British Poultry Science, Edinburgh, v. 33, n. 1, p. 201206, 1992. 